International Mathematical Forum, 4, 2009, no. 24, 1157 - 1171

\title{
A Generalized Eigenmode Algorithm for Reducible Regular Matrices over the Max-Plus Algebra
}

\author{
Zvi Retchkiman Königsberg \\ Instituto Politécnico Nacional, CIC \\ Mineria 17-2, Col. Escandon, Mexico D.F 11800, Mexico \\ mzvi@cic.ipn.mx
}

\begin{abstract}
In this paper an algorithm for computing a generalized eigenmode of reducible regular matrices over the max-plus algebra is proposed. Given a matrix of finite size, the problem consists in giving an algorithm which will tell us how to compute its generalized eigenmode over the max plus algebra. The solution to the problem is achieved by studying some type of recurrent equations. In fact, by transforming the reducible regular matrix into its normal form, and considering a very specific recurrent equation, an explicit mathematical characterization is obtained, upon which the algorithm is constructed.
\end{abstract}

Mathematics Subject Classification: 08C99, 16Y60, 65F15, 05C50, 15A29, 15A33

Keywords: Max-Plus Algebra, Reducible Matrices, Eigenmode, Recurrent Equations, Algorithm

\section{INTRODUCTION}

In this paper an algorithm for computing a generalized eigenmode of reducible regular matrices over the max-plus algebra is proposed. Given a matrix of finite size such that in each one of its rows has at least one finite element and whose communication graph is not strongly connected, the problem consists in giving an algorithm which will tell us how to compute its generalized eigenmode over the max plus algebra, which indeed has an idempotent semiring, or also called dioid, mathematical structure. The notion of generalized eigenmode, as its name says, results to be a genaralization of the notion of eigenvalues 
and eigenvectors for the case when the matrix under study is irreducible i.e., has a communication graph which is strongly connected. The solution to the problem is achieved by studying some type of recurrent equations. In fact, by transforming the reducible regular matrix into a block upper triangular form, called normal form, and considering a very specific recurrent equation, an explicit mathematical characterization is obtained, upon which the algorithm is constructed. The theoretical mathematical foundation exposed in the paper was taken from ([1] and [2]) incorporating the author's personal style and trying to improve its statements, as well as its proofs, whenever it was possible. The main idea of the algorithm was inspired by ([2] and the references quoted therein) and up to the author's knowledge, it has not been exploded and completely developed as is done in this paper before. Another alternative algorithm for computing a generalized eigenmode of a reducible and regular matrix, is Howard's algorithm which is based on a policy iteration improvement procedure which in numerical examples has proven to be very efficient,(see [3]). What is nice from the algorithm discussed in this work is its mathematical foundation. The paper is organized as follows. In section 2 , the concept of max-plus algebra is defined, its algebraic structure is also described. Matrices and graphs are presented, the spectral theory of matrices is discussed, finally the problem of solving linear equations is addressed. Section 3, starts by introducing the concept of generalized eigenmode. Once this has been done, it continues by discussing, how to compute the generalized eigenmode for recurrent equations for the cases of irreducible and reducible

matrices. In section 4, the algorithm is formally presented, two illustrative examples are also included. Finally, in section 5, some conclusions are given.

\section{MAX-PLUS ALGEBRAS}

In this section the concept of max-plus algebra is defined. Its algebraic structure is described. Matrices and graphs are presented. The spectral theory of matrices is discussed. Finally the problem of solving linear equations is addressed.

\subsection{Basic Definitions}

NOTATION: $\mathbb{N}$ is the set of natural numbers, $\mathbb{R}$ is the set of real numbers, $\epsilon=-\infty, e=0, \mathbb{R}_{\max }=\mathbb{R} \cup\{\epsilon\}, \underline{n}=1,2, \ldots, n$

Let $a, b \in \mathbb{R}_{\max }$ and define the operations $\oplus$ and $\otimes$ by:

$$
a \oplus b=\max (a, b) \text { and } a \otimes b=a+b .
$$

(Notice that: $a \oplus \epsilon=\epsilon \oplus a=a$ and $a \otimes e=e \otimes a=a, \forall a \in \mathbb{R}_{\max }$.) 
Definition 1 The set $\mathbb{R}_{\max }$ with the two operations $\oplus$ and $\otimes$ is called a maxplus algebra and is denoted by $\Re_{\max }=\left(\mathbb{R}_{\max }, \oplus, \otimes, \epsilon, e\right)$.

Definition 2 A semiring is a nonempty set $R$ endowed with two operations $\oplus_{R}, \otimes_{R}$, and two elements $\epsilon_{R}$ and $e_{R}$ such that:

- $\oplus_{R}$ is associative and commutative with zero element $\epsilon_{R}$;

- $\otimes_{R}$ is associative, distributes over $\oplus_{R}$, and has unit element $e_{R}$,

- $\epsilon_{R}$ is absorbing for $\otimes_{R}$ i.e., $a \otimes_{R} \epsilon=\epsilon_{R} \otimes a=a, \forall a \in R$.

Such a semiring is denoted by $\Re=\left(R, \oplus_{R}, \otimes_{R}, \epsilon, e\right)$. In addition if $\otimes_{R}$ is commutative then $R$ is called a commutative semiring, and if $\oplus_{R}$ is such that $a \oplus_{R} a=a, \forall a \in R$ then it is called idempotent.

Theorem 3 The max-plus algebra $\Re_{\max }=\left(\mathbb{R}_{\max }, \oplus, \otimes, \epsilon, e\right)$ has the algebraic structure of a commutative and idempotent semiring.

Proof. The proof follows immediately using the definitions given by equation (1) (in a similar way to the case for addition and multiplication over the reals) just being careful when one substitutes multiplication for the max operation. As for example in the distributive property for $a, b, c \in \mathbb{R}_{\max }$, it holds that: $a \otimes(b \oplus c)=a+\max (b, c)=\max (a+b, a+c)=(a \otimes b) \oplus(a \otimes c)$.

\subsection{Matrices and Graphs}

Let $\mathbb{R}_{\max }^{n \times n}$ be the set of $n \times n$ matrices with coefficients in $\mathbb{R}_{\max }$ with the following operations:

- The sum of matrices $A, B \in \mathbb{R}_{\max }^{n \times n}$, denoted $A \oplus B$ is defined by:

$$
(A \oplus B)_{i j}=a_{i j} \oplus b_{i j}=\max \left(a_{i j}, b_{i j}\right)
$$

for $i$ and $j \in \underline{n}$.

- The product of matrices $A \in \mathbb{R}_{\max }^{n \times l}, B \in \mathbb{R}_{\max }^{l \times n}$, denoted $A \otimes B$ is defined by:

$$
(A \oplus B)_{i k}=\bigoplus_{j=1}^{l} a_{i j} \otimes b_{j k}=\max _{j \in \underline{l}}\left\{a_{i j}+b_{j k}\right\}
$$

for $i$ and $k \in \underline{n}$. (Notice that the matrix product in general fails to be commutative.) 
- The scalar product for $\alpha \in \mathbb{R}_{\max }^{n}$ and $A \in \mathbb{R}_{\max }^{n \times n}$, denoted $\alpha \otimes A$ is defined by:

$$
(\alpha \oplus A)_{i j}=\alpha \otimes a_{i j}
$$

for $i$ and $j \in \underline{n}$.

Let $\mathcal{E} \in \mathbb{R}_{\max }^{n \times n}$ denote the matrix with all its elements equal to $\epsilon$ and denote by $E \in \mathbb{R}_{\max }^{n \times n}$ the matrix which has its diagonal elements equal to $e$ and all the other elements equal to $\epsilon$. Then, the following result, whose proof is immediate, can be stated.

Theorem 4 The 5-tuple $\Re_{\max }^{n \times n}=\left(\mathbb{R}_{\text {max }}^{n \times n}, \oplus, \otimes, \mathcal{E}, E\right)$ has the algebraic structure of a noncommutative idempotent semiring.

Definition 5 Let $A \in \mathbb{R}_{\max }^{n \times n}$ and $k \in \mathbb{N}$ then the $k$-th power of $A$ denoted by $A^{\otimes k}$ is defined by:

$$
A^{\otimes k}=\underbrace{A \otimes A \otimes \cdots \otimes A}_{k-\text { times }}
$$

where $A^{\otimes 0}$ is set equal to $E$.

Definition 6 A matrix $A \in \mathbb{R}_{\max }^{n \times n}$ is said to be regular if $A$ contains at least one element distinct from $\epsilon$ in each row.

Next, an overview in the theory of graphs will be given, emphasizing the rich relationship that exist between them and matrices.

Definition 7 Let $\mathcal{N}$ be a finite and non-empty set and consider $\mathcal{D} \subseteq \mathcal{N} \times \mathcal{N}$. The pair $G=(\mathcal{N}, \mathcal{D})$ is called a directed graph, where $\mathcal{N}$ is the set of elements called nodes and $\mathcal{D}$ is the set of ordered pairs of nodes called arcs. A directed graph $G=(\mathcal{N}, \mathcal{D})$ is called a weighted graph if a weight $w(i, j) \in \mathbb{R}$ is associated with any $\operatorname{arc}(i, j) \in \mathcal{D}$.

Let $A \in \mathbb{R}_{\max }^{n \times n}$ be any matrix, a graph $\mathcal{G}(A)$, called the communication graph of $A$, can be associated as follows. Define $\mathcal{N}(A)=\underline{n}$ and a pair $(i, j) \in \underline{n} \times \underline{n}$ will be a member of $\mathcal{D}(A) \Leftrightarrow a_{j i} \neq \epsilon$, where $\mathcal{D}(A)$ denotes the set of arcs of $\mathcal{G}(A)$.

Definition 8 A path from node $i$ to node $j$ is a sequence of arcs $p=\left\{\left(i_{k}, j_{k}\right) \in\right.$ $\mathcal{D}(A)\}_{k \in \underline{m}}$ such that $i=i_{1}, j_{k}=i_{k+1}$, for $k<m$ and $j_{m}=j$. The path $p$ consists of the nodes $i=i_{1}, i_{2}, \ldots, i_{m}, j_{m}=j$ with length $m$ denoted by $|p|_{1}=$ $m$. In the case when $i=j$ the path is said to be a circuit. A circuit is said to be elementary if nodes $i_{k}$ and $i_{l}$ are different for $k \neq l$. A circuit consisting of one arc is called a self-loop. 
Let us denote by $P(i, j ; m)$ the set of all paths from node $i$ to node $j$ of length $m \geq 1$ and for any $\operatorname{arc}(i, j) \in \mathcal{D}(A)$ let its weight be given by $a_{i j}$ then the weight of a path $p \in P(i, j ; m)$ denoted by $|p|_{w}$ is defined to be the sum of the weights of all the arcs that belong to the path. The average weight of a path $p$ is given by $|p|_{w} /|p|_{1}$. Given two paths, as for example, $p=$ $\left(\left(i_{1}, i_{2}\right),\left(i_{2}, i_{3}\right)\right)$ and $q=\left(\left(i_{3}, i_{4}\right),\left(\left(i_{4}, i_{5}\right)\right.\right.$ in $\mathcal{G}(A)$ the concatenation of paths $\circ: \mathcal{G}(A) \times \mathcal{G}(A) \rightarrow \mathcal{G}(A)$ is defined as $p \circ q=\left(\left(i_{1}, i_{2}\right),\left(i_{2}, i_{3}\right),\left(i_{3}, i_{4}\right),\left(i_{4}, i_{5}\right)\right)$. The communication graph $\mathcal{G}(A)$ and powers of matrix $A$ are closely related as it is shown in the next theorem, whose proof follows using induction on the length $k$ of the path (see [1]).

Theorem 9 Let $A \in \mathbb{R}_{\max }^{n \times n}$, then $\forall k \geq 1$ :

$$
\left[A^{\otimes k}\right]_{j i}=\max \left\{|p|_{w}: p \in P(i, j ; k)\right\}
$$

where $\left[A^{\otimes k}\right]_{j i}=\epsilon$ in the case when $P(i, j ; k)$ is empty i.e., no path of length $k$ from node $i$ to node $j$ exists in $\mathcal{G}(A)$.

Definition 10 Let $A \in \mathbb{R}_{\max }^{n \times n}$ then define the matrix $A^{+} \in \mathbb{R}_{\max }^{n \times n}$ as:

$$
A^{+}=\bigoplus_{k=1}^{\infty} A^{\otimes k}
$$

sometimes known as the shortest path matrix. Where the element $\left[A^{+}\right]_{j i}$ gives the maximal weight of any path from $j$ to $i$. If in addition one wants to add the possibility of staying at a node then one must include matrix $E$ in the definition of matrix $A^{+}$giving rise to its Kleene star representation defined by:

$$
A^{*}=\bigoplus_{k=0}^{\infty} A^{\otimes k}
$$

Lemma 11 Let $A \in \mathbb{R}_{\max }^{n \times n}$ be such that any circuit in $\mathcal{G}(A)$ has average circuit weight less than or equal to $\epsilon$. Then it holds that:

$$
A^{*}=\bigoplus_{k=0}^{n-1} A^{\otimes k} .
$$

Proof. Since $A^{*}=\bigoplus_{k=0}^{\infty} A^{\otimes k}=\left(\bigoplus_{k=0}^{n-1} A^{\otimes k}\right) \oplus\left(\bigoplus_{k \geq n}^{\infty} A^{\otimes k}\right)$ and all paths of length greater than or equal to $n$ are made up of a circuit and a path of length strictly less than $n$, we have that $A^{k} \leq A \oplus A^{* 2} \oplus \cdots \oplus A^{*(n-1)} \forall k \geq n$, which implies that $A^{*}=\bigoplus_{k=0}^{n-1} A^{\otimes k}$. 
Definition 12 Let $G=(\mathcal{N}, \mathcal{D})$ be a graph and $i, j \in \mathcal{N}$, node $j$ is reachable from node $i$, denoted as $i \mathcal{R} j$, if there exists a path from $i$ to $j$. A graph $G$ is said to be strongly connected if $\forall i, j \in \mathcal{N}, j \mathcal{R} i . A$ matrix $A \in \mathbb{R}_{\max }^{n \times n}$ is called irreducible if its communication graph is strongly connected, when this is not the case matrix $A$ is called reducible.

Definition 13 Let $G=(\mathcal{N}, \mathcal{D})$ be a not strongly connected graph and $i, j \in$ $\mathcal{N}$, node $j$ communicates with node $i$, denoted as $i \mathcal{C} j$, if either $i=j$ or $i \mathcal{R} j$ and $j \mathcal{R} i$.

The relation $i \mathcal{C} j$ defines an equivalence relation in the set of nodes, and therefore a partition of $\mathcal{N}$ into a disjoint union of subsets, the equivalence classes, $\mathcal{N}_{1}, \mathcal{N}_{2}, \ldots, \mathcal{N}_{q}$ such that $\mathcal{N}=\mathcal{N}_{1} \cup \mathcal{N}_{2} \cup \ldots \cup \mathcal{N}_{q}$ or $\mathcal{N}=\bigcup_{i \in \mathcal{N}}[i]$; $[i]=\{j \in \mathcal{N}: i C j\}$.

Given the above partition, it is possible to focus on subgraphs of $G$ denoted by $G_{r}=\left(\mathcal{N}_{r}, \mathcal{D}_{r}\right) ; r \in \underline{q}$ where $\mathcal{D}_{r}$ denotes the subset of arcs, which belong to $\mathcal{D}$, that have both the begin node and end node in $\mathcal{N}_{r}$. If $\mathcal{D}_{r} \neq \varnothing$, the subgraph $G_{r}=\left(\mathcal{N}_{r}, \mathcal{D}_{r}\right)$ is known as a maximal strongly connected subgraph of $G$.

Remark 14 In case of having an isolated node $i$ (i.e., a node that does not communicate with any other node) and which does not even have an arc from it to itself, the associated subgraph is given by $([i], \varnothing)$ which is not strongly connected however, for convenience it will be considered as if it were.

Definition 15 The reduced graph $\widetilde{G}=(\widetilde{\mathcal{N}}, \widetilde{\mathcal{D}})$ of $G$ is defined by setting $\widetilde{\mathcal{N}}=$ $\left\{\left[i_{1}\right],\left[i_{2}\right], \ldots\left[i_{q}\right]\right\}$ and $\left(\left[i_{r}\right],\left[i_{s}\right]\right) \in \widetilde{\mathcal{D}}$ if $r \neq s$ and there exists an arc $(k, l) \in \mathcal{D}$ for some $k \in\left[i_{r}\right]$ and $l \in\left[i_{s}\right]$.

let $A_{r r}$ denote the matrix by restricting $A$ to the nodes in $\left[i_{r}\right] \forall r \in \underline{q}$ i.e., $\left[A_{r r}\right]_{k l}=a_{k l} \forall k, l \in\left[i_{r}\right]$. Then $\forall r \in \underline{q}$ either $A_{r r}$ is irreducible or is equal to $\epsilon$. Therefore since by construction the reduced graph does not contain any circuits, the original reducible matrix $A$ after a possible relabeling of the nodes in $G(A)$, can be written as:

$$
A=\left(\begin{array}{ccccc}
A_{11} & A_{12} & \cdots & \cdots & A_{1 q} \\
\mathcal{E} & A_{22} & \cdots & \cdots & A_{2 q} \\
\mathcal{E} & \mathcal{E} & A_{33} & & \vdots \\
\vdots & \vdots & \ddots & \ddots & \vdots \\
\mathcal{E} & \mathcal{E} & \cdots & \mathcal{E} & A_{q q}
\end{array}\right)
$$

with matrices $A_{s r} 1 \leq s<r \leq q$ of suitable size, where each finite entry in $A_{s r}$ corresponds to an arc from a node in $\left[i_{r}\right]$ to a node in $\left[i_{s}\right]$.

Definition 16 Let $A \in \mathbb{R}_{\max }^{n \times n}$ be a reducible matrix then, the block upper triangular given by (10) is said to be a normal form of matrix $A$. 


\subsubsection{Spectral Theory}

Definition 17 Let $A \in \mathbb{R}_{\max }^{n \times n}$ be a matrix. If $\mu \in R_{\max }$ is a scalar and $v \in$ $R_{\text {max }}^{n}$ is a vector that contains at least one finite element such that:

$$
A \otimes v=\mu \otimes v
$$

then, $\mu$ is called an eigenvalue and $v$ an eigenvector.

Remark 18 Notice that the eigenvalue can be equal to $\epsilon$ and is not necessarily unique. Eigenvectors are certainly not unique indeed, if $v$ is an eigenvector then $\alpha \otimes v$ is also an eigenvector for all $\alpha \in \mathbb{R}$.

Let $\mathcal{C}(A)$ denote the set of all elementary circuits in $\mathcal{G}(A)$ and write:

$$
\lambda=\max _{p \in \mathcal{C}(A)} \frac{|p|_{w}}{|p|_{1}}
$$

for the maximal average circuit weight. Notice that $\operatorname{since} \mathcal{C}(A)$ is a finite set, the maximum of (12) is attained (which is always the case when matrix $A$ is irreducible). In case $\mathcal{C}(A)=\emptyset$ define $\lambda=\epsilon$.

Definition $19 A$ circuit $p \in G(A)$ is said to be critical if its average weight is maximal. The critical graph of $A$, denoted by $G^{c}(A)=\left(\mathcal{N}^{c}(A), \mathcal{D}^{c}(A)\right)$, is the graph consisting of those nodes and arcs that belong to critical circuits in $G(A)$.

Lemma 20 Let assume that $G(A)$ contains at least one circuit then, any circuit in $G^{c}(A)$ is critical.

Proof. If this were not the case, we could find a circuit $p \in G^{c}(A)$, composed of sub-paths, lets say $p_{i}$ of critical circuits $p^{c}$, with weight different from $\lambda$ (which without loss of generality will be assumed to be equal to $e$ ). If this circuit had a weight greater than $e$ then, since $p$ is also a circuit in $G(A)$, it would contradict the assumption that the maximal average circuit weight $\lambda$ is equal to $e$. On the other hand, if the weight of it were less than $e$, since the maximal average circuit weight is $\lambda=e$, the circuit composed of the union of the complements of the paths $p_{i} \in G^{c}(A)$, with respect to $G(A)$, must have positive weight, in order to assure that the critical circuits $p^{c} \in G(A)$, to which the sub-paths $p_{i}$ belong is critical i.e., has average wight $\lambda=\epsilon$, which is also a contradiction. Therefore, any circuit in $G^{c}(A)$ is critical.

Definition 21 Let $A \in \mathbb{R}_{\max }^{n \times n}$ be a matrix and $\mu$ an eigenvalue of $A$ with associated eigenvector $v$ then, the support of $v$ consists of the set of nodes of $G(A)$ which correspond to finite entries of $v$. 
Lemma 22 Let $A \in \mathbb{R}_{\max }^{n \times n}$ be an irreducible matrix then any $v \in R_{\max }^{n}$ which satisfies (11) has all components different from $\epsilon$.

Proof. Let us assume that the support of $v$ does not cover the whole node set of $G(A)$ then since $A$ is irreducible, there are arcs going from nodes in the support of $v$ going to nodes not belonging to the support of $v$ i.e., there exists a node $j$ in the support of $v$ and a node $i$ not in the support of $v$ with $a_{i j} \neq \epsilon$. But this implies that $[A \otimes v]_{i} \geq a_{i j} \otimes v_{j}>\epsilon$ therefore, the support of $A \otimes v$ is larger than the support of $v$ which contradicts (11).

Next, the most important result of this sub-section is given.

Theorem 23 If $A \in \mathbb{R}_{\max }^{n \times n}$ is irreducible, then there exists one and only one finite eigenvalue (with possible several eigenvectors). This eigenvalue is equal to the maximal average weight of circuits in $G(A)$ :

$$
\lambda(A)=\max _{p \in \mathcal{C}(A)} \frac{|p|_{w}}{|p|_{1}}
$$

Proof. Existence of the eigenvalue $\lambda$ and the eigenvector $v$. Consider matrix $A_{\lambda}$ with elements $\left[A_{\lambda}\right]_{i j}=a_{i j}-\lambda, \lambda$ finite. The maximum average circuit of $A_{\lambda}$ is $e$. Hence, lemma (11) implies that $A_{\lambda}^{*}$ and $A_{\lambda}^{+}$exist. Moreover, from lemma (20), matrix $A_{\lambda}^{+}$is such that that $\forall \eta \in \mathcal{N}^{c}(A):\left[A_{\lambda}^{+}\right]_{\eta \eta}=e$. Let $[A]_{. k}$ denote the $k$ th column of matrix $A$ then, since $\forall \eta \in \mathcal{N}^{c}(A):\left[A_{\lambda}^{+}\right]_{\eta \eta}=$ $e \Rightarrow\left[A_{\lambda}^{*}\right]_{\eta \eta}=e+\left[A_{\lambda}^{+}\right]_{\eta \eta}=e$, it follows that $\left[A_{\lambda}^{+}\right]_{. \eta}=\left[A_{\lambda}^{*}\right]_{. \eta}$. But $A_{\lambda}^{+}=A_{\lambda} \oplus A_{\lambda}^{*}$ which implies that:

$$
\left[A_{\lambda} \oplus A_{\lambda}^{*}\right]_{. \eta}=\left[A_{\lambda}^{*}\right]_{. \eta} \Rightarrow A_{\lambda} \oplus\left[A_{\lambda}^{*}\right]_{. \eta}=\left[A_{\lambda}^{*}\right]_{. \eta} \Longleftrightarrow A \oplus\left[A_{\lambda}^{*}\right]_{. \eta}=\lambda \oplus\left[A_{\lambda}^{*}\right]_{. \eta} .
$$

Hence, it follows that $\lambda$ is an eigenvalue of matrix $A$ with associated eigenvector $v$ the $\eta$ th column of $A_{\lambda}^{*}$ for all $\eta \in \mathcal{N}^{c}(A)$.

Uniqueness. Suppose $\mu \neq \lambda$ satisfies (11) and pick any circuit $\gamma=\left(\left(\eta_{1}, \eta_{2}\right),\left(\eta_{2}, \eta_{3}\right)\right.$ $\left., \ldots,\left(\eta_{l}, \eta_{l+1}\right)\right) \in G(A)$ of length $l=|\gamma|_{1}$ with $\eta_{l+1}=\eta_{1}$. Then, since $a_{\eta_{k+1} \eta_{k}} \neq \epsilon$ with $k \in \underline{l}$, it follows that $a_{\eta_{k+1} \eta_{k}} \oplus v_{\eta_{k}} \leq \mu \oplus v_{\eta_{k+1}}, k \in \underline{l}$, where lemma (22) assures that all components of $v \neq \epsilon$, but this implies that $\bigotimes_{k=1}^{l} a_{\eta_{k+1} \eta_{k}} \oplus v_{\eta_{k}} \leq \mu^{\otimes l} \oplus \bigotimes_{k=1}^{l} v_{\eta_{k+1}}$ which in conventional algebra can be written as: $\sum_{k=1}^{l} a_{\eta_{k+1} \eta_{k}}+v_{\eta_{k}} \leq \mu \times l+\sum_{k=1}^{l} v_{\eta_{k+1}}$ which is reduced to $\sum_{k=1}^{l} a_{\eta_{k+1} \eta_{k}} \leq \mu \times l$ or $|\gamma|_{W} \leq \mu \times l \Rightarrow \frac{|\gamma|_{W}}{|\gamma|_{l}} \leq \mu$. But since this holds for every circuit in $G(A) \mu$ has to be equal to $\lambda$.

\subsubsection{Linear Equations}

Theorem 24 Let $A \in \mathbb{R}_{\max }^{n \times n}$ and $b \in \mathbb{R}_{\text {max }}^{n}$. If the communication graph $G(A)$ has maximal average circuit weight less than or equal to $e$, then $x=A^{*} \otimes b$ 
solves the equation $x=(A \otimes x) \oplus b$. Moreover, if the circuit weights in $G(a)$ are negative then, the solution is unique.

Proof. Existence. By lemma (11) $A^{*}$ exists. Substituting the proposed solution into the equation one gets:

$$
\begin{gathered}
x=\left(A \otimes\left[A^{*} \otimes b\right]\right) \oplus b=\left(A \otimes A^{*} \otimes b\right) \oplus(e \oplus b)= \\
{\left[\left(A \otimes A^{*}\right) \oplus e\right] \otimes b=\left[A \otimes A^{*}\right] \oplus b=A^{*} \oplus b .}
\end{gathered}
$$

Uniqueness. Let $y$ be another solution of $x=(A \otimes x) \oplus b$ then substituting $y=b \oplus(A \otimes y)$ it follows that: $y=b \oplus(A \otimes b) \oplus\left(A^{\otimes 2} \otimes y\right)$, iterating once and once again, one gets: $y=b \oplus(A \otimes b) \oplus\left(A^{\otimes 2} \otimes b\right) \oplus \ldots \oplus\left(A^{\otimes(k-1)} \otimes b\right) \oplus\left(A^{\otimes k} \otimes y\right)=$ $\left[\bigoplus_{l=0}^{k-1}\left(A^{\otimes l} \otimes b\right)\right] \otimes\left(A^{\oplus k} \oplus y\right)$. Now, since by assumption circuits have negative weight the right side of the above equation, as $k$ goes to $\infty$ tend to $\mathcal{E}$ while the left side, using lemma (11), tends to $A^{*} \otimes b$ therefore, $y=x$.

\section{GENERALIZED EIGENMODE AND RE- CURRENT EQUATIONS}

This section starts by introducing the concept of generalized eigenmode. Once this has been done, the section continues by discussing, how to compute the generalized eigenmode for recurrent equations for the cases of irreducible and reducible matrices.

Definition 25 Let $A \in \mathbb{R}_{\max }^{n \times n}$ be a regular matrix, a pair of vectors $(\eta, v) \in$ $\mathbb{R}^{n} \times \mathbb{R}^{n}$ is called a generalized eigenmode of $A$ if for all $k \geq 0$ :

$$
A \oplus(k \times \eta+v)=(k+1) \times \eta+v
$$

Remark 26 It is important to underline that the second vector $v$ in a generalized eigenmode is not unique. Indeed, if $(\eta, v)$ is a generalized eigenmode then the pair $(\eta, \nu \oplus v) \forall \nu \in \mathbb{R}$, also works.

Theorem 27 Consider the inhomogeneous recurrent equation

$$
x(k+1)=A \otimes x(k) \oplus \bigoplus_{j=1}^{m} B_{j} \otimes u_{j}(k), k \geq 0
$$

with $A \in \mathbb{R}_{\text {max }}^{n \times n}$ irreducible with eigenvalue $\lambda=\lambda(A)$, or $A \in \mathbb{R}_{\max } A=\epsilon$ with $\lambda=\epsilon,\left\{B_{j}\right\}_{j=1}^{m} \in \mathbb{R}_{\max }^{n \times m_{j}}$ for some appropriate $m_{j} \geq 1$ matrices different 
from $\mathcal{E}, u_{j}(k) \in \mathbb{R}^{m_{j}}$ such that $u_{j}(k)=w_{j}(k) \otimes \tau_{j}^{\otimes k}, k \geq 0$, with $\tau_{j} \in \mathbb{R}$ and $w_{j} \in \mathbb{R}^{m_{j}}$. Denote $\tau=\bigoplus_{j \in \underline{m}} \tau_{j}$. Then, there exists an integer $K \geq 0$ and $a$ vector $v \in \mathbb{R}^{n}$ such that the sequence $x(k)=v \otimes \mu^{\otimes k}$ with $\mu=\lambda \otimes \tau$ satisfies equation (15) for all $k \geq K$.

Proof. The proof is given by considering two possible cases.

Case $\lambda>\tau$. Since $A$ is irreducible, theorem (23) and lemma (22), guarantee the existence of the eigenvalue $\lambda$ with associated finite eigenvector $v \in \mathbb{R}^{n}$. Choose $v$ such that $v \oplus \lambda>\bigoplus_{j=1}^{m} B_{j} \otimes w_{j}$, this can always be done since if not, it is possible to replace $v$ by $v \otimes \rho, \rho$ an arbitrary but fixed real number which can be picked as big as desired (see remark (18)). Set $\mu=\lambda>\tau_{j} \forall j \in \underline{m}$ then, $\forall k \geq 0$ it follows that: $v \otimes \mu^{\otimes(k+1)}=A \otimes v \otimes \mu^{\otimes k}$ and since $\mu^{\otimes(k+1)} \geq \bigoplus_{j=1}^{m} B_{j} \otimes w_{j} \otimes \tau_{j}^{\otimes k}$ it implies that $v \otimes \mu^{\otimes(k+1)}=A \otimes v \otimes \mu^{\otimes k} \oplus \bigoplus_{j=1}^{m} B_{j} \otimes w_{j} \otimes \tau_{j}^{\otimes k}$. Therefore, equation (15) is satisfied $\forall k \geq 0$.

Case $\lambda \leq \tau$.

Sub-case (1): $A$ is a matrix. Recall that $\tau=\bigoplus_{j \in \underline{m}} \tau_{j}$ and assume that the maximum is attained by the first $r \tau^{\prime}$ s, which can always be accomplished by a proper renumbering of the sequences $u_{j}(k), j \in \underline{m}$. Now, look at the equation:

$$
s=A_{\tau} \otimes s \oplus \bigoplus_{j=1}^{r}\left(B_{j}\right)_{\tau} \otimes w_{j}
$$

where $A_{\tau}$ and $\left(B_{j}\right)_{\tau}, j \in \underline{m}$ are obtained from their original matrices $A$ and $\left(B_{j}\right)$ by subtracting $\tau$ from all of its finite elements. Because $\lambda \leq \tau$, the communication graph of $A_{\tau}$ only contains circuits with a non-positive weight therefore, from theorem $(24)$ a solution $v$ exists, further since $\left(A_{\tau}\right)^{*}$ is completely finite $\left(A_{\tau}\right.$ is strongly connected) and $\bigoplus_{j=1}^{r}\left(B_{j}\right)_{\tau} \otimes w_{j}$ contains at least one finite element it implies that $v$ is finite i.e., $v \in \mathbb{R}^{n}$. But this implies that;

$$
v \otimes \tau=A \otimes v \oplus \bigoplus_{j=1}^{r} B_{j} \otimes w_{j}
$$

Then, setting $\mu=\tau=\tau_{j}, j=1,2, \ldots, r$ it follows that:

$$
v \otimes \mu^{\otimes(k+1)}=A \otimes v \otimes \mu^{\otimes k} \oplus \bigoplus_{j=1}^{r} B_{j} \otimes w_{j} \otimes \tau_{j}^{\otimes k}, \forall k \geq 0
$$


wich leads to:

$$
v \otimes \mu^{\otimes(k+1)} \leq A \otimes v \otimes \mu^{\otimes k} \oplus \bigoplus_{j=1}^{m} B_{j} \otimes w_{j} \otimes \tau_{j}^{\otimes k} .
$$

However since $\mu>\tau_{j}$ for $j=r+1, r+2, \ldots, m$, there exists an integer $K \geq 0$, as large as needed such that $\forall k \geq K v \otimes \mu^{\otimes(k+1)} \geq \bigoplus_{j=r+1}^{m} B_{j} \otimes w_{j} \otimes \tau_{j}^{\otimes k}$. Therefore, equation (15) is satisfied $\forall k \geq K$.

Sub-case (2): $A$ is the scalar $\epsilon$ with $\lambda=\epsilon$. Take $v$, solution of (16), as $v=\bigoplus_{j=1}^{r}\left(B_{j}\right)_{\tau} \otimes w_{j}$ and proceed exactly as it was done in sub-case (1).

Remark 28 Notice that in theorem (27) equation (15) is satisfied for all $k \geq K$. However, in the case where it is possible to reinitialize the sequences $u_{j}(k)=w_{j}(k) \otimes \tau_{j}^{\otimes k}, k \geq 0$, by redefining the vectors $w_{j}$ for $j \in$ $\underline{m}$ then, it is possible to satisfy equation (15) $\forall k \geq 0$. Indeed, just set $v=v \otimes \mu^{\otimes K}, w_{j}(k)=w_{j}(k) \otimes \tau_{j}^{\otimes K}, j \in \underline{m}$. Then, the new sequences $x(k)=v \otimes \mu^{\otimes k}, u_{j}(k)=w_{j}(k) \otimes \tau_{j}^{\otimes k} j \in \underline{m}$ solve our problem $\forall k \geq 0$.

Now, let us consider the recurrent equation:

$$
x(k+1)=A \otimes x(k), k \geq 0
$$

with $A$ reducible and regular. Recalling what was presented in sub-section (2.2) (see also definition (16)), and using that matrix $A$ is regular, it follows that matrix $A$ can always be rewritten in its normal form i.e.,

$$
A=\left(\begin{array}{ccccc}
A_{11} & A_{12} & \cdots & \cdots & A_{1 q} \\
\mathcal{E} & A_{22} & \cdots & \cdots & A_{2 q} \\
\mathcal{E} & \mathcal{E} & A_{33} & & \vdots \\
\vdots & \vdots & \ddots & \ddots & \vdots \\
\mathcal{E} & \mathcal{E} & \cdots & \mathcal{E} & A_{q q}
\end{array}\right)
$$

with the conditions that $A_{q q}$ is irreducible, that for $i \in \underline{q-1}$ either $A_{i i}$ is an irreducible matrix or is equal to $\epsilon$, and that the $A_{i j}$ matrices are different from $\mathcal{E}$ for $i, j=i+1 ; i \in \underline{q}$. Let the vector $x(k)$ be partitioned according to the normal form given by equation (18) as:

$$
x(k)=\left(\begin{array}{c}
x_{1}(k) \\
x_{2}(k) \\
\vdots \\
x_{q}(k)
\end{array}\right)
$$


where $x_{i}(k), i \in \underline{q}$ are vectors of suitable size. Therefore the recurrent equation given by equation (17) can be written as:

$$
x(k+1)=A_{i i} \otimes x_{i}(k) \oplus \bigoplus_{j=1+1}^{q} A_{i j} \otimes x_{j}(k) ; i \in \underline{q}, k \geq 0
$$

Then, the next result follows.

Theorem 29 Consider the recurrent equation given by equation (19). Assume that $A_{q q}$ is irreducible and that for $i \in q-1$ either $A_{i i}$ is an irreducible matrix or is equal to $\epsilon$. Assume also, that the $A_{i j}$ matrices are different from $\mathcal{E}$ for $i, j=i+1 ; i \in \underline{q}$. Then, there exist finite vectors $v_{1}, v_{2}, \ldots, v_{q}$ of suitable size and scalars $\xi_{1}, \xi_{2}, \ldots, \xi_{q} \in \mathbb{R}$ such that the sequences:

$$
x_{i}(k)=v_{i} \otimes \xi_{i}^{\otimes k}, i \in \underline{q}
$$

satisfy equation (19) for all $k \geq 0$. The scalars $\xi_{1}, \xi_{2}, \ldots, \xi_{q} \in \mathbb{R}$ are determined by:

$$
\xi_{i}=\bigoplus_{j \in \mathcal{H}_{i}} \xi_{j} \oplus \lambda_{i}
$$

where $\mathcal{H}_{i}=\left\{j \in \underline{q}: j>i, A_{i j} \neq \mathcal{E}\right\}$.

Proof. The proof follows straightforward by first considering the case $i=q$, for which the result is immediate, and then proceeding backwards step by step. Using, at each step, the result given by theorem (27), whose hypothesis are automatically satisfied. The fact that the theorem holds $\forall k \geq 0$ follows since all the sequences $x_{i}(k) \in \underline{q}$ can be reinitialized, see remark (28).

Corollary 30 Let $A \in \mathbb{R}_{\max }^{n \times n}$ be a reducible and regular matrix, then there exist a pair of vectors $(\eta, v) \in \mathbb{R}^{n} \times \mathbb{R}^{n}$, a generalized eigenmode, such that for all $k \geq 0$ :

$$
A \oplus(k \times \eta+v)=(k+1) \times \eta+v
$$

Proof. From what was discussed above theorem (29) about reducible and regular matrices, and applying it. The pair $\eta=\left(\xi_{1}, \xi_{2}, \ldots, \xi_{n}\right) \in \mathbb{R}^{n}, v=$ $\left(v_{1}, v_{2}, \ldots, v_{q}\right) \in \mathbb{R}^{n}$ result to be a generalized eigenmode which satisfies (20) for all $k \geq 0$.

The result provided by corollary (30) plays a fundamental role in the proposed algorithm for reducible matrices, as will be seen in the next section. 


\section{AN ALGORITHM FOR COMPUTING GEN- ERALIZED EIGENMODES OF REDUCIBLE MATRICES}

This section illustrates how by means of theorems $(27,29)$ and corollary $(30)$, an algorithm for computing a generalized eigenmode for reducible matrices can be proposed. Two numerical examples are included, (see [1]).

\section{Algorithm}

1. Take $A \in \mathbb{R}_{\max }^{n \times n}$ a reducible and regular matrix.

2. Using the material presented in (2.2) bring it to the normal form and write it in the form of system (19).

3. Consider the last equation of system (19) i.e., the $n$th equation, and compute its eigenvalue $\lambda_{n}$ with associated eigenvector $v_{n}$, set $\xi_{n}=\lambda_{n}$ and $j=n$.

4. Consider the above next $(j-1)$ th equation, and compute the eigenvalue of matrix $A_{(j-1)(j-1)}$, called it $\lambda_{j-1}$.

5. Is $\lambda_{j-1}>\xi_{j}$, if this is the case go to 6 if not, go to 7 .

6. Set $\xi_{j-1}=\lambda_{j-1}$ and compute $v_{j-1}$ according to the first case of the proof of theorem (27). Go to 8.

7. Set $\xi_{j-1}=\xi_{j}$ and compute $v_{j-1}$ according to the second case of the proof of theorem $(27)$.

8. Decrease $j$ by one. Is $j \neq 1$ go back to 4 if not finish.

At the end the algorithm provides one pair of vectors $\eta=\left(\xi_{1}, \xi_{2}, \ldots, \xi_{n}\right) \in \mathbb{R}^{n}$, $v=\left(v_{1}, v_{2}, \ldots, v_{q}\right) \in \mathbb{R}^{n}$ which result to be a generalized eigenmode of matrix $A \in \mathbb{R}_{\max }^{n \times n}$.

Remark 31 Theorem (23) can be used for computing the eigenvalues of the irreducible matrices $\left\{A_{i i} ; i \in \underline{n}\right\}$. In addition, the power algorithm (see [1]) results of great help for computing the eigenvector in case it comes from the solution of equation (11).

Example 32 Consider the following regular reducible matrix already in its normal form:

$$
A=\left(\begin{array}{llll}
1 & 2 & \varepsilon & 7 \\
\varepsilon & 3 & 5 & \varepsilon \\
\varepsilon & 4 & \varepsilon & 3 \\
\varepsilon & 2 & 8 & \varepsilon
\end{array}\right)
$$


with $A_{11}=1$, and $A_{22}=\left(\begin{array}{ccc}3 & 5 & \varepsilon \\ 4 & \varepsilon & 3 \\ 2 & 8 & \varepsilon\end{array}\right)$.

From $A_{22}$ we get that $\lambda_{2}=\max \{10 / 3,11 / 2,9 / 2,3\}=11 / 2=\xi_{2}$ and using the power algorithm or doing algebra that $v_{2}=(20,41 / 2,23)$. Now, since $A_{11}=1$ this implies that $\lambda_{1}=1 \leq \xi_{2}$ therefore $\xi_{1}=\xi_{2}=11 / 2$ and $v_{1}=49 / 2$ is obtained as the solution of $\left(1 \otimes v_{1}\right) \oplus 22 \oplus 30=11 / 2 \otimes v_{1}$. Therefore, the pair $\eta=(11 / 2,11 / 2,11 / 2,11 / 2), v=(49 / 2,20,41 / 2,23)$ results to be $a$ generalized eigenmode. Notice that subtracting 21 to each member of $v$ we get that $\eta=(11 / 2,11 / 2,11 / 2,11 / 2), v=(6 / 2,-1,-1 / 2,2)$ is also a generalized eigenmode

Example 33 Consider the following regular reducible matrix:

$$
\left(\begin{array}{cccccccccc}
\varepsilon & 0 & \varepsilon & \varepsilon & \varepsilon & \varepsilon & \varepsilon & \varepsilon & \varepsilon & \varepsilon \\
\varepsilon & \varepsilon & -3 & \varepsilon & \varepsilon & \varepsilon & \varepsilon & \varepsilon & \varepsilon & \varepsilon \\
\varepsilon & 4 & \varepsilon & 0 & \varepsilon & \varepsilon & \varepsilon & \varepsilon & \varepsilon & \varepsilon \\
0 & \varepsilon & \varepsilon & \varepsilon & \varepsilon & \varepsilon & \varepsilon & \varepsilon & \varepsilon & \varepsilon \\
\varepsilon & 16 & \varepsilon & \varepsilon & \varepsilon & -5 & \varepsilon & \varepsilon & \varepsilon & \varepsilon \\
\varepsilon & \varepsilon & \varepsilon & \varepsilon & \varepsilon & \varepsilon & 0 & \varepsilon & \varepsilon & \varepsilon \\
\varepsilon & \varepsilon & \varepsilon & \varepsilon & 9 & \varepsilon & \varepsilon & \varepsilon & \varepsilon & \varepsilon \\
\varepsilon & \varepsilon & \varepsilon & \varepsilon & \varepsilon & \varepsilon & 1 / 2 & \varepsilon & \varepsilon & \varepsilon \\
\varepsilon & \varepsilon & \varepsilon & \varepsilon & \varepsilon & 6 & \varepsilon & \varepsilon & \varepsilon & \varepsilon \\
9 & \varepsilon & \varepsilon & \varepsilon & \varepsilon & \varepsilon & \varepsilon & \varepsilon & \varepsilon & \varepsilon
\end{array}\right)
$$

The communication graph $\mathcal{G}(\mathcal{A})$ has five maximal strongly connected subgraphs which implies that its reduced graph, $\widetilde{G}=(\widetilde{\mathcal{N}}, \widetilde{\mathcal{D}})$ turns out to be defined by: $\widetilde{\mathcal{N}}=\{[1],[5],[8],[9],[10]\}, \widetilde{\mathcal{D}}=\{([1],[10]),([1],[5]),([5],[8]),([5],[9])\}$, where $[1]=\{1,2,3,4\},[5]=\{5,6,7\},[8]=\{8\},[9]=\{9\}$, and $[10]=\{10\}$. Based on the reduced graph, after placing the rows and columns of matrix $A$ in the order $8,9,5,6,7,10,1,2,3,4$ the following normal form of matrix $A$ is obtained:

$$
\left(\begin{array}{cccccccccc}
\varepsilon & \varepsilon & \varepsilon & \varepsilon & 1 / 2 & \varepsilon & \varepsilon & \varepsilon & \varepsilon & \varepsilon \\
\varepsilon & \varepsilon & \varepsilon & 6 & \varepsilon & \varepsilon & \varepsilon & \varepsilon & \varepsilon & \varepsilon \\
\varepsilon & \varepsilon & \varepsilon & -5 & \varepsilon & \varepsilon & \varepsilon & 16 & \varepsilon & \varepsilon \\
\varepsilon & \varepsilon & \varepsilon & \varepsilon & 0 & \varepsilon & \varepsilon & \varepsilon & \varepsilon & \varepsilon \\
\varepsilon & \varepsilon & 9 & \varepsilon & \varepsilon & \varepsilon & \varepsilon & \varepsilon & \varepsilon & \varepsilon \\
\varepsilon & \varepsilon & \varepsilon & \varepsilon & \varepsilon & \varepsilon & 9 & \varepsilon & \varepsilon & \varepsilon \\
\varepsilon & \varepsilon & \varepsilon & \varepsilon & \varepsilon & \varepsilon & \varepsilon & 0 & \varepsilon & \varepsilon \\
\varepsilon & \varepsilon & \varepsilon & \varepsilon & \varepsilon & \varepsilon & \varepsilon & \varepsilon & -3 & \varepsilon \\
\varepsilon & \varepsilon & \varepsilon & \varepsilon & \varepsilon & 6 & \varepsilon & 4 & \varepsilon & 0 \\
\varepsilon & \varepsilon & \varepsilon & \varepsilon & \varepsilon & \varepsilon & 0 & \varepsilon & \varepsilon & \varepsilon
\end{array}\right)
$$


with $A_{11}=A_{22}=A_{44}=\epsilon, A_{33}=\left(\begin{array}{ccc}\varepsilon & -5 & \varepsilon \\ \varepsilon & \varepsilon & 0 \\ 9 & \varepsilon & \varepsilon\end{array}\right)$ and $A_{55}=\left(\begin{array}{cccc}\varepsilon & 0 & \varepsilon & \varepsilon \\ \varepsilon & \varepsilon & -3 & \varepsilon \\ \varepsilon & 4 & \varepsilon & 0 \\ 0 & \varepsilon & \varepsilon & \varepsilon\end{array}\right)$.

From $A_{55}$ we get that $\lambda_{5}=\max \{1 / 2,-3 / 4\}=1 / 2=\xi_{5}$ and doing algebra that $v_{5}=(17 / 2,9,25 / 2,8)$. Now, since $A_{11}=\epsilon$ this implies that $\lambda_{1}=\epsilon \leq \xi_{2}$ therefore $\xi_{4}=\xi_{5}=1 / 2$ and that $v_{1}=17$. Proceeding with $A_{33}$ we get that $\lambda_{3}=4 / 3>\xi_{4}$ therefore, we obtain that $\xi_{3}=4 / 3$ and that $v_{3}=(24,91 / 3,95 / 3)$, which is obtained from the solution of $A_{33} \otimes v_{3}=\lambda_{3} \otimes v_{3}$ and $\lambda_{3} \otimes v_{3_{2}}>a_{38} \otimes v_{5_{2}}=25$. Iterating one more time, we get for $A_{22}$ that $\xi_{2}=4 / 3$ and $v_{2}=35$. An finally, for $A_{11}, \xi_{1}=4 / 3$ and $v_{1}=185 / 6$ Therefore, the pair $\eta=(4 / 3,4 / 3,4 / 3,4 / 3,4 / 3,1 / 2,1 / 2,1 / 2,1 / 2,1 / 2), v=$ $(185 / 6,35,24,91 / 3,95 / 3,17,17 / 2,9,25 / 2,8)$ results to be a generalized eigenmode.

\section{CONCLUSIONS}

This work gives an algorithm for computing a generalized eigenmode of reducible regular matrices over the max-plus algebra. Given a reducible regular matrix, the first step consists in, transforming it into its normal form. Once this has been done the following steps are constructed based on an explicit mathematical characterization, which comes out to be a consequence of considering a very specific recurrent equation.

\section{References}

[1] B. Heidergott, G. J. Olsder, J. van der Woude, Max Plus at Work, Princeton University Press, 2006.

[2] F. Baccelli, G. Cohen, G. J. Olsder, J. P. Quadrat, Synchronization and Linearity, Web-edition, 2001.

[3] C. Terrason, G. Cohen, S. Gaubert, M. McGettrick, J. P. Quadrat. "Numerical computation of spectral elements in max-plus algebra". Proceedings of the IFAC conference in Systems Structure and Control. Nantes, France, 1998.

Received: September, 2008 